\title{
A VISCOELASTIC MODEL OF THE HUMAN SPINE SUBJECTED TO $+\boldsymbol{g}_{z}$ ACCELERATIONS*
}

\author{
C. T. TERRY \\ Office of Surgeon General, Research and Development Command, Washington, D.C. \\ and \\ VERNE L. ROBERTS \\ Highway Safety Research Institute, The University of Michigan, Ann Arbor, Michigan
}

\begin{abstract}
A viscoelastic rod model which includes damping has been used to simulate the spinal column mathematically. This one-dimensional rod was subjected to a ramp input acceleration and the theoretical acceleration at the end of the rod was compared to the experimental head acceleration data from previously conducted cadaver tésts. The agreement between the experimental and theoretical results is better than for the elastic rod model previously published, but indicates further nonlinear considerations are still needed.
\end{abstract}

\section{INTRODUCTION}

THE NEED for an accurate mathematical model of the spine subjected to $+g_{z}$ accelerations is apparent from a review of the problems involved in the seat ejection of pilots from aircraft (Levy, 1964). The prediction of spinal failure due to acceleration can be accomplished either experimentally through a statistically representative series of destructive volunteer tests, or mathematically by using subtolerance level testing of volunteers or cadavers to provide a model for mathematical extrapolation. The mathematical model is more feasible and should be developed on the assumption that it would act similarly to the spine in all responses, e.g. accelerations and stresses. The model should be constructed so as to correlate with experimental subtolerance response and then used to obtain accurate predictions of the response up to the physical limits of the system.

Latham (1957) developed the first mathematical model to describe the dynamic response of the spinal column to $+g_{z}$ accelerations. His model was a simple dynamic system (Fig. 1) which was correlated with experimental data resulting from vibration studies. One of the obvious limitations of his model was that the accelerations could be found only at the endpoints (head and hip), whereas the probable

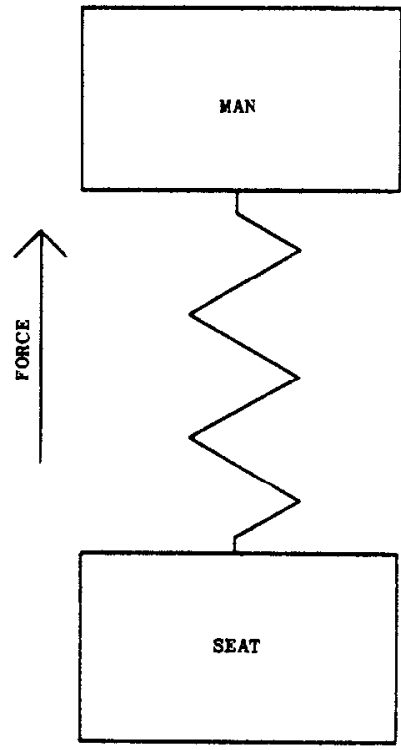

Fig. 1. Lathams model of the spinal column.

area of injury lies along the spinal column. This single-degree-of-freedom system model could not produce a dynamic load factor greater than two, which was in fact obtained experimentally by Lissner and Evans (1963), and Hodgson et al. (1963).

Stech (1963) also represented the spinal column with a spring-mass system by adding the experimental spring constants (Yorra, 1963) of all the vertebra in series and arriving

*Received 7 May 1967. 
at a composite figure for the whole spine. With this analysis the frequency of the body in the spinal mode $(\sim 6 \mathrm{~Hz})$ was calculated and found to agree with Coermann's impedance data (Coermann, 1961). However, his model actually describes the whole body in the spinal mode rather than the desired model of the spinal column itself.

Hess and Lombard (1958) were the first to look at the spinal column as a continuum. They simulated the spine with a continuous elastic rod and compared mathematically derived accelerations with some experimental ones at different points along the spine. The correlation between the elastic rod analogy and experimental results was fairly good (Fig. 2) for the initial pulse response, but

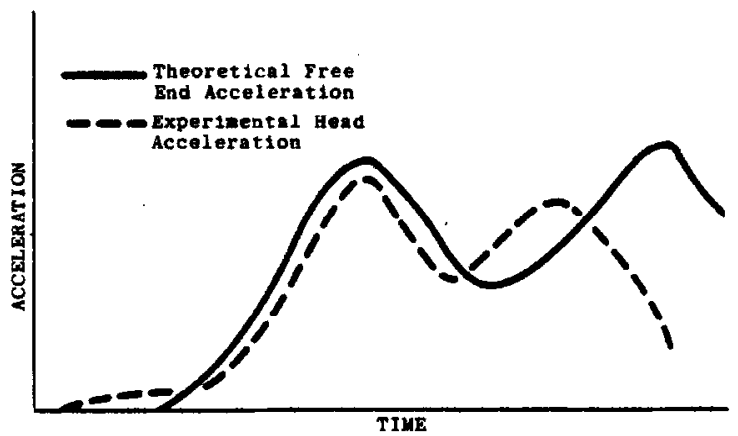

Fig. 2. Hess and Lombard's correlation of theoretical elastic rod and spinal column accelerations.

the need for some inherent damping was immediately apparent. In this paper a viscoelastic model of the spine is proposed which would include the required damping, and would accurately reproduce the experimental accelerations.

\section{MATHEMATICAL MODEL}

The model chosen for representation of the spinal column was a uniform rod of a Maxwell-type viscoelastic medium. To check the results of such a model, the accelerations at some point on the theoretical rod will be checked against some experimental accelerations at the same corresponding point on the spine. The theoretical model was subjected to a ramp input acceleration pulse at one end to conform to the experimental acceleration input (Fig. 3).

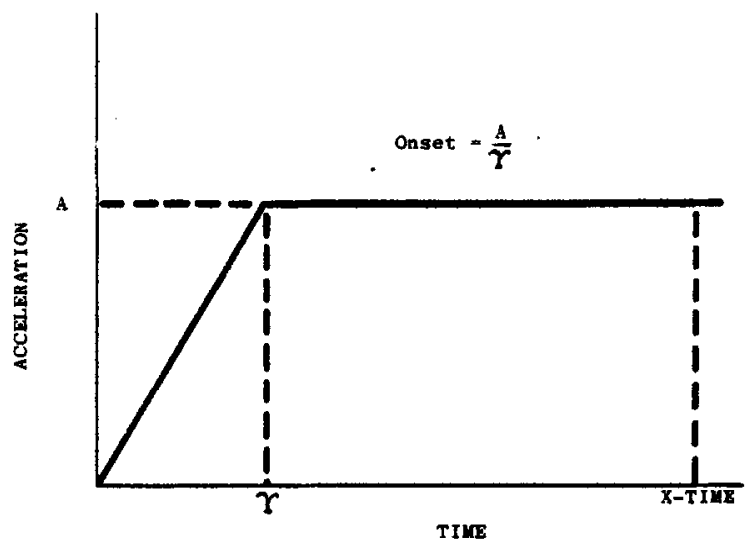

Fig. 3. A typical ramp input.

The analysis of the resulting acceleration at the far end of the rod, which corresponds with the experimental head acceleration, is as follows:

The equation of motion of a one-dimensional wave in a uniform rod is

By definition

$$
\begin{gathered}
\sigma_{x}=\rho U_{t t} . \\
\epsilon=U_{x} .
\end{gathered}
$$

The constitutive equation for a Maxwell-type material is

$$
\epsilon_{t}=(1 / E) \sigma_{t}+\mu \sigma .
$$

Combining equations (2) and (3) to eliminate $\epsilon$, then differentiating this new equation and equation (1) with respect to $t$ and rearranging the results into a compatible form yields

$$
\begin{aligned}
a_{t} & =(1 / \rho) S_{x} \\
a_{x} & =(1 / E) S_{t}+\mu S .
\end{aligned}
$$

These equations are recognized as partial differential equations of the hyperbolic type. The method of characteristics was used to solve this system of quasi-linear equations (Lister, 1964). To expedite the solution, this method of calculation was programmed for 
the IBM 7074 with the following boundary conditions

$$
\begin{array}{lll}
a(o, t)=(A / Y) t & t \leq Y & x=0 \\
a(o, t)=A & t \geq Y & x=0 \\
S(L, t)=0 & & x=L .
\end{array}
$$

The program was written so that for ramp inputs with different values of $A$ and $Y$, the acceleration at the far end of the rod (analogous to the acceleration of the head) could be determined.

In order for the evaluation of the problem to be complete, some values must be assigned to $E$ and $\mu$. The modulus of elasticity of bone has been fairly well documented for static tests; however, as pointed out by McElhaney and Byars (1965), the modulus of elasticity is a function of the strain rate in dynamic tests. If the elastic model is used as a guide to arrive at a value of $E$, a simple calculation can be performed. Hess and Lombard (1958) obtained the best correlation when the time required for the acceleration wave to travel the length of the model was about $0.025 \mathrm{sec}$.
Assuming the spine is $2.5 \mathrm{ft}$ in length and the time of travel is $0.025 \mathrm{sec}$, the resulting wave velocity is $100 \mathrm{ft} / \mathrm{sec}$. If this velocity is used for the elastic rod, then $E / \rho$ must be of the order of $10^{4} \mathrm{ft}^{2} / \mathrm{sec}^{2}(V=\sqrt{E / \rho})$. However, assuming the density of bone to be 3.6 slugs $/ \mathrm{ft}^{3}$ yields a value of $E$ of approximately $3.6 \times 10^{4}$ $\mathrm{lb} / \mathrm{ft}^{2}$, which does not compare favorably with the accepted value of $E$ for bone; consequently it can be assumed that the intervertebral disc plays the major role in the transmission of the acceleration waves (assuming the wave velocity is about $100 \mathrm{ft} / \mathrm{sec}$ ). For this reason the initial value of $E$ selected was $3.6 \times 10^{*}$ $\mathrm{lb} / \mathrm{ft}^{2}$, while the value of the viscosity $(1 / \mu)$, principally a function of the intervertebral disc, was $\sim 10^{6} \mathrm{P}$.

Once the values of $E$ and $\mu$ were selected. the computer program iterated the values of the constants in each test to optimize the acceleration response of the theoretical viscoelastic rod.

The theoretical curves for two of the ten sets of data are shown with the corresponding experimental curves in Figs. 4 and 5 . Indicated

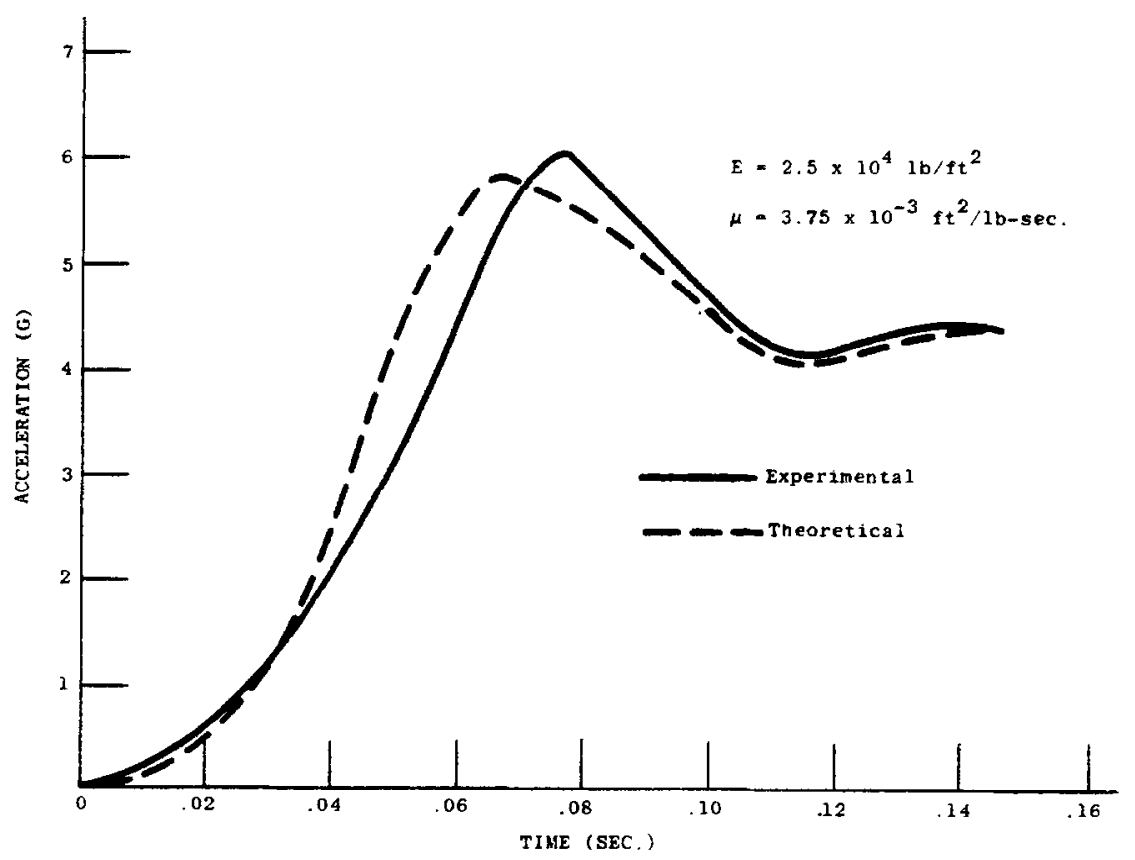

Fig. 4. Low level acceleration correlation of viscoelastic rod and spinal column. 


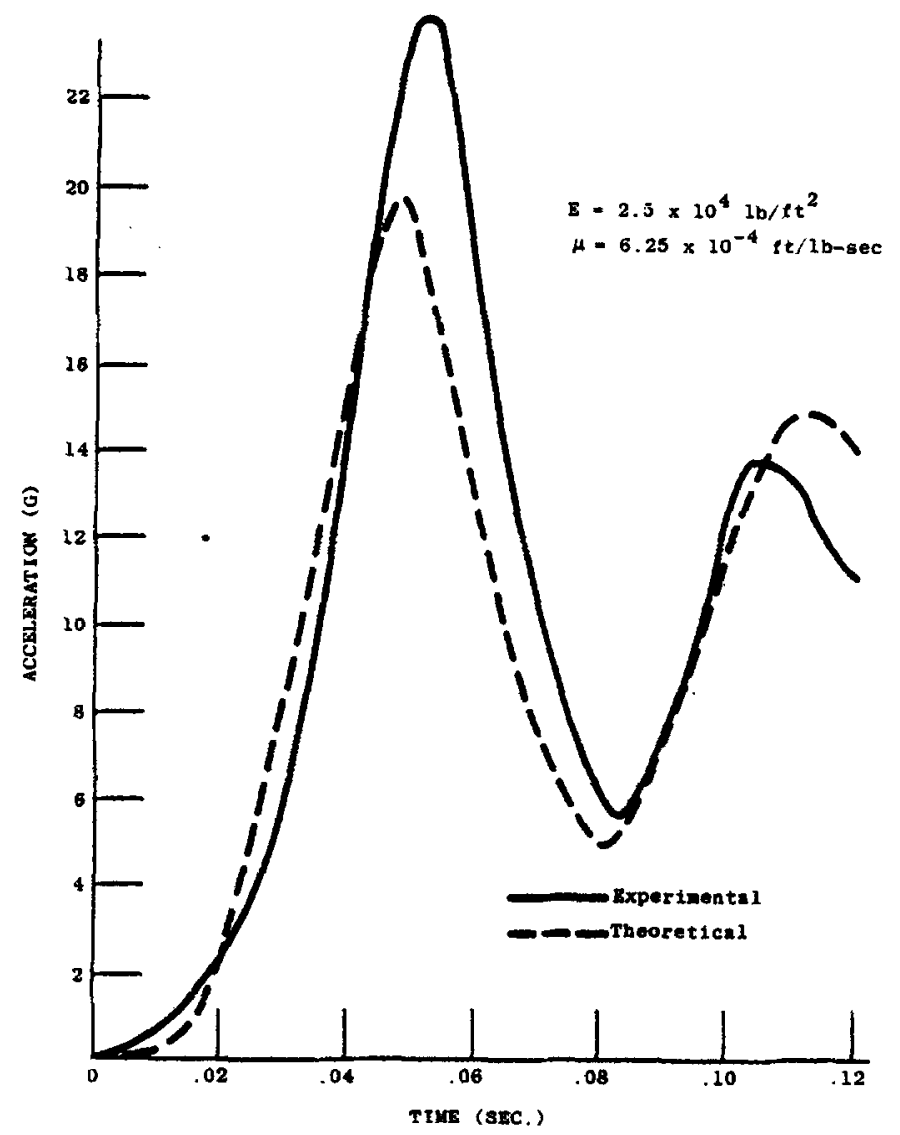

Fig. 5. High level acceleration correlation of viscoelastic rod and spinal column.

on each set of curves are the values of $E$ and $\mu$ which gave the overall best fit between the experimental and theoretical. Figures 6 and 7 illustrate graphically how $E$ varied with both rate of onset and acceleration and how $\mu$ varied with onset rate. It should be pointed out that the "best" values were those that fit the overall curve the best. In the computer program the error between the curves was calculated as the difference of area between the curves divided by the area of the experimental curve. This error was then minimized by adjusting the constants in order to give the "best" values of these constants. From a comparison of the theoretical acceleration curves and the experimental head accelerations, the following results can be noted:
(1) The values of the theoretical curves closely match the experimental ones for the set of data with low acceleration level $(4 \cdot 5 \mathrm{~g})$.

(2) As the acceleration level increased, the difference between the theoretical and experimental curve also increased, particularly at the first peak for the values.

(3) It was evident that the computed response frequency of the theoretical model was a constant, while the experimental results yielded curves at which no set frequency could satisfy all the peaks.

(4) By adjusting the constants $E$ and $\mu$, the theoretical peak acceleration could be made to exceed twice the value of the steadystate input acceleration as required by the experimental data. 


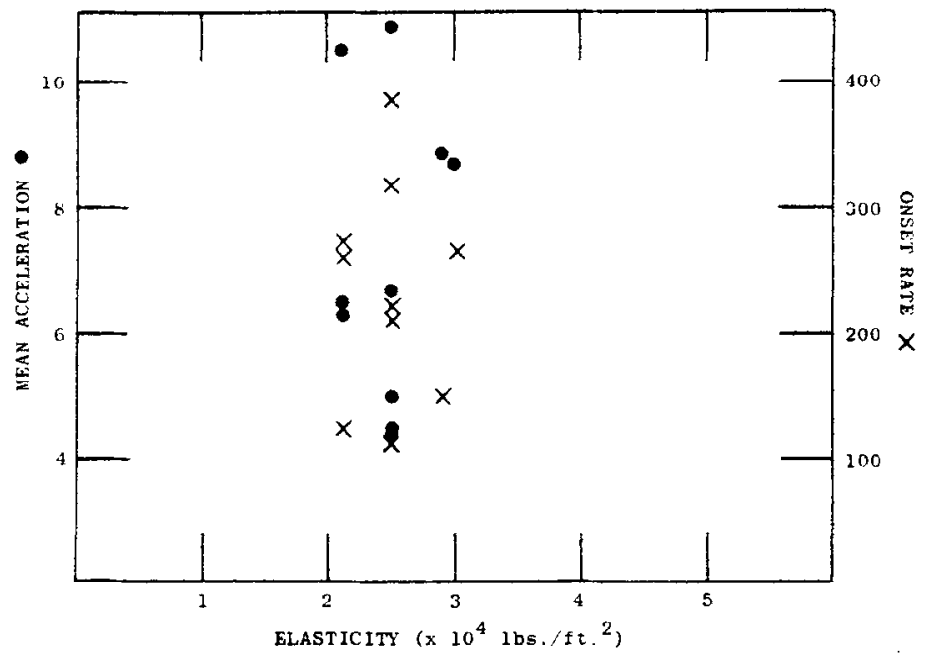

Fig. 6. Variation of elasticity with mean acceleration and onset rate.

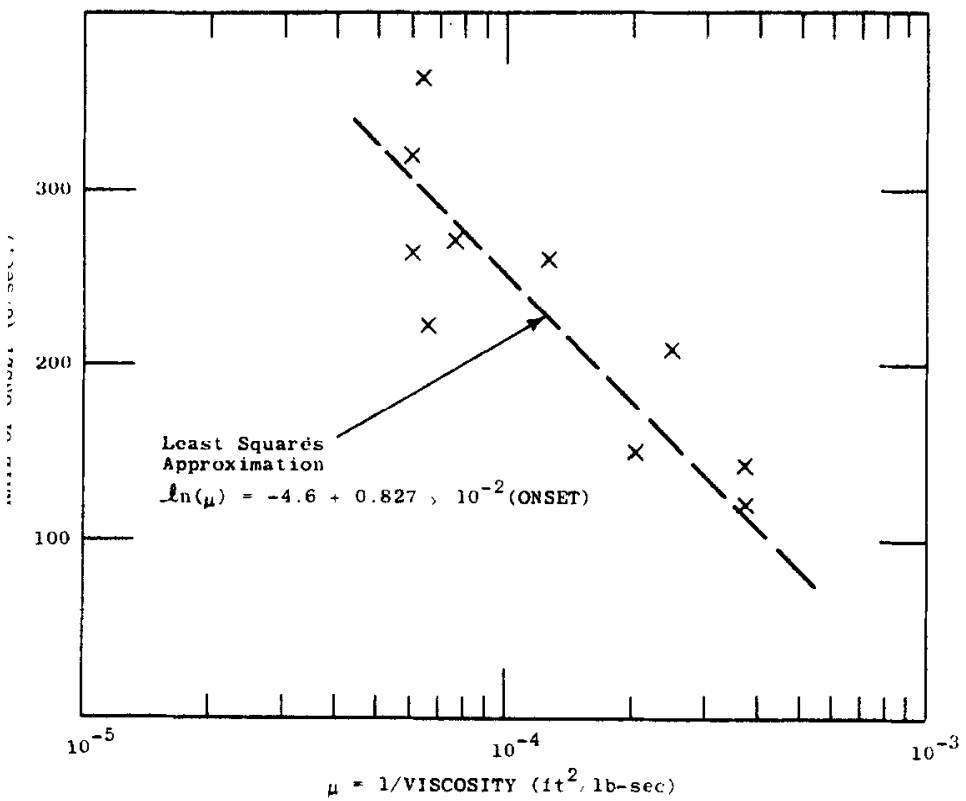

Fig. 7. Variation of viscosity with rate of onset. 


\section{DISCUSSION}

A possible explanation for the discrepancies noted in the comparison of results is presented in the same order as in the list of discrepancies.

(1) A close inspection of the comparison of the curves shows a small time lag in the initial peak of the theoretical curve. However, the rest of the curve matches fairly well. The reason for the match lies in the fact that the acceleration levels do not vary too much between the first and the second peak for each of these low level tests, and thus any dependence of the constants upon strain rate would probably not be too noticeable.

(2) The discrepancy of the first peak becomes more obvious for the higher levels of acceleration. A factor contributing to the gap between the first peaks was the unreliability of the input ramp function as the level of acceleration increased. The most frequent variance from the ideal ramp function was a slight "overshoot" of the mean acceleration at the end of the ascending portion of the ramp. This would tend to increase the level of acceleration at the first peak. It should be pointed out at this time that it was possible to match the first peak by greatly reducing the viscosity $(1 / \mu)$. However, when this was done the theoretical peaks following the first were considerably higher than the experimental. The total error as calculated above became very great for such a matching and this method was abandoned. Such a procedure might be used when considering effective damping of the model leading up to the first peak where the strain rate is the highest. This would slow down the theoretical response for this period and result in a more closely matched phase at the first peak. During the subsequent response, when the strain rate becomes less, the damping would be decreased, resulting in a speed-up of the theoretical response which would more closely match the experimental and theoretical acceleration curves (see Figs. 4 and 5). Of course, the validity of such superposition could only be proven by a new set of equations in which the dependence of $\mu$ upon strain rate had been considered initially. Whether this set of equations would support the above analysis is only speculative. As can be seen from Fig. 6 , the elasticity was essentially independent of the acceleration level or onset rate. This result agrees basically with Hess and Lombard's data from live tests of ejection seats. It is obvious, though, that if the frequency response is made to depend more upon the viscous element, the elasticity too will change, depending on how greatly the viscous element affects the frequency response and the levels of strain rate involved. In effect, this will make the elasticity, too, dependent on the strain rate, as indicated by McElhaney and Byars (1965).

\section{CONCLUSIONS}

From an inspection of Figs. 4 and 5, it can be concluded that the overall response of the viscoelastic rod more closely approximates the true acceleration response than does the elastic rod analogy of Hess and Lombard (1958), and that the use of the Maxwell element is not out of the question as they indicated. In Fig. 8 a response of the Maxwell model for a complete acceleration input is shown compared with the experimental head acceleration.

For the following reasons, no attempt was made to use the calculated accelerations along the rod to predict tolerance to acceleration:

(1) The most significant accelerations would probably be those occurring at the first peak of acceleration response. As was discussed previously, the theoretical response tended to be lower at this first peak. In order to predict stress, which will be the governing physical input determining the limits, a reliable relation between the stress and acceleration at any point must be known. The body forces acting on the vertebra should be considered, but this would involve an estimation of the body force on each segment, which has not been documented at this time. 


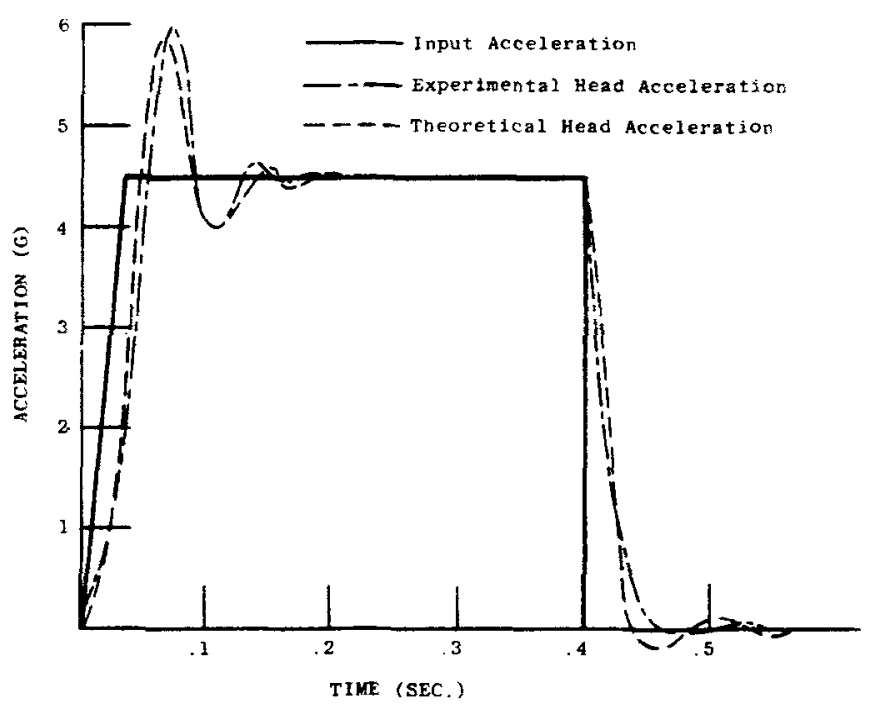

Fig. 8. Complete response history of low level acceleration correlation.

However, it is obvious that if the acceleration response is not accurate at the critical points, then the stress will also be unreliable. Therefore, until a better correlation can occur at the first peak, a tolerance curve would not be accurate.

(2) Another reason for omitting a tolerance curve is the lack of data concerning the end point failures for each vertebra and intervertebral disc. Most investigators have used the basic work of Ruff (1950) without question. But, as noted by McElhaney and Byars (1965), the point of failure for bone depends upon the strain rate. Therefore, it is obvious that the lack of knowledge of material properties of the vertebral column is a significant roadblock to determining a tolerance curve.

From Fig. 6 it can be seen that the average elasticity for accurate frequency response was $2.5 \times 10^{4} \mathrm{lb} / \mathrm{ft}^{2}$. This value of elasticity yields a wave velocity of approximately $83 \mathrm{ft} / \mathrm{sec}$. For a spinal column of $2.5 \mathrm{ft}$, this yields a travel time of $0.03 \mathrm{sec}$. This compares favorably with Hess and Lombard's (1958) value of $0.025 \mathrm{sec}$ obtained from live tests.

\section{RECOMMENDATIONS}

The results obtained using the Maxwell model approximation justify some modifications. The first modification of the theory could be to use a viscous element in which the viscosity is a function of the strain rate. This would permit the nonlinear response dictated by the experimental results. and. in effect, would also render elasticity dependent upon strain rate. Of course, any changes in the basic theory would also affect the method of solution, and as the sophistication of the theory increases so do the complications arising in the method of solution. The next viscoelastic element which could be considered as a model would be the Kelvin element. This process of modifying model after model could continue until a discrete number of mass-spring-dashpot elementsone for each individual vertebra-could be considered. But the best model will be the one which combines maximum accuracy with maximum simplicity.

As more sophisticated models are developed, more complex experimental data will be required to adequately describe in- 
herent physical constants. Much work needs to be done on the rheological properties 'of the vertebra and the intervertebral disc. Such experimentation could determine how the viscous and elastic constants vary with the strain rate or other input parameters. A wellplanned program of experiments could definitely shed light on the qualitative and quantitative behavior of the individual components of the spinal column.

\section{REFERENCES}

Coerman, Rolf R. (1961) The mechanical impedance of the human body in sitting and standing position at low frequencies, $A S D$ Tech. Rep. 61-492. WrightPărterson AFB.

Hess, J. L. and Lombard, C. V. (1958) Theoretical investigations of dynamic response of man to high vertical accelerations, J. Aviat. Med. 29, 66.

Hodgson, V. R., Lissner, H. R. and Patrick, L. M. (1963) The effect of jerk on the human spine, ASME Paper No. 63-WA.316.

Latham, F. (1957) A study of body ballistics: seat ejection, Proc. R. Soc. B 147, 121.

Levy, Phillip M. (1964) Ejection seat design and vertebral fractures. J. Aerospace Med. 35 (6), 545.

Lissner, H. R. and Evans, F. G. (1963) Effects of acceleration on the human skeleton. Report to Public Health Service Grant AC-00054-06.

Lister, M. (1964) The numerical solution of hyperbolic partial differential equations by the method of characteristics. Mathematical Methods For Digizal Computers, (Edited by A. Ralston and H. S. Wili) pp. 165179. Wiley, New York.
McElhaney, J. H. and Byars, E. F. (1965) Dynamic response of biological materials. ASME Paper 65-WA/HUF-9.

Ruff, Siegfried (1950) Brief acceleration: less than one second. German Aviation Medicine-World War II. Vol. 1, Chap. VI-C, Dept. Air Force, 584-599.

Stech, E. L. (1963) Calculation of human spinal frequency from cadaver data and comparison with tests on live human subjects. Report 122-100, Frost Engineering Development Corporation.

Yorra, A. J. (1963) The investigation of the structural behavior of the intervertebral disc. Master's Thesis M.I.T.

\section{NOMENCLATURE}

$A$ plateau acceleration on ramp input

a instantaneous acceleration

$a_{t} \partial a / \partial t$

$a_{x} \partial \alpha / \partial x$

$\epsilon$ strain

$\epsilon_{t} \quad \partial \epsilon / \partial t$

$\stackrel{\epsilon_{t}}{E}$ modulus of elasticity

$L$ length of viscoelastic rod (spinal column)

$1 / \mu$ viscosity

$\rho$ density

$\sigma$ stress

$\sigma_{t} \quad \partial \sigma / \partial t$

$\sigma_{x} \partial \sigma / \partial x$

$S$ do/at stress rate

$S_{t} \partial^{2} \sigma \partial \partial t^{2}$

$S_{x} \partial^{2} \sigma / \partial i \partial x$

0 particle deplacement

$U_{x} \partial U / \partial x$ strain

$U_{t t} \partial^{2} U / \partial t^{2}$ acceleration

$t$ time

$Y$ rise time for ramp input

$x$ distance from input end of viscoelastic rod or hip end of spinal column 